\title{
Sensitivity of Stratospheric Forecasts to Tropospheric Forecast
}

\author{
By Carlos R. Mechoso and Akio Arakawa \\ Department of Atmospheric Sciences, University of California, \\ Los Angeles. Los Angeles, CA 90024 \\ (Manuscript received 5 November 1986; in revised form 12 February 1987)
}

\begin{abstract}
The sensitivity of stratospheric forecasts to tropospheric forecasts is investigated using the UCLA GCM. For this purpose, forecasts for the major stratospheric sudden warming of February 1979 are compared to "forecasts" from identical initial conditions but with selected components of the tropospheric fields periodically replaced by the observed. The comparison shows that the accuracy of stratospheric forecasts can be drastically improved when errors in the tropospheric forecast, particularly in the zonal mean flow, are reduced.
\end{abstract}

\section{Introduction}

Major sudden warmings are remarkable events in the winter circulation of the Northern Hemisphere stratosphere. During these events, increases in high-latitude temperatures and reversals of the flow occur in planetary-scale during a few day period. This combination of space and time scales is revealing of the major role played by dynamical processes in the stratosphere.

Since the pioneering study of Miyakoda et al. (1970), it has been shown that general circulation models (GCMs) can predict stratospheric warmings from several days in advance (Simmons and Strüfing, 1983; Mechoso et al., 1985). GCMs can then be used to perform controlled experiments in order to elucidate crucial contributions to the success in the prediction and thereby to the development of the phenomenon.

Mechoso et al. (1985) investigated the predictability of stratospheric warming events during the winter of 1979 by performing a series of 10-day forecasts with the low- and high-resolution versions of the 15-layer UCLA GCM. Their forecasts were highly success- ful for some cases and less successful for other cases. In the case from 15 February, 10-day forecasts were poor with only marginal improvement for increased horizontal resolution. Rapidly increasing errors in the refractive index for wavenumber 2 , the dominant planetary wave during the period, were found in the upper troposphere during the early part of these forecasts. In the case from 17 February, on the other hand, the 10-day forecast with the high horizontal resolution was highly successful while that with the low horizontal resolution was not. Significant differences in the refractive index for wavenumber 2 between the forecasts were found in the upper troposphere during the second half of the 10-day period. Based on these findings and their implications for vertical propagation of planetary waves, Mechoso et al. (1985) interpreted that the success of stratospheric forecasts largely depends on that of tropospheric forecasts, particularly in the zonal mean flow.

The objective of this study is to test the validity of the above interpretation. Our approach consists in comparing forecasts from 15 and 17 February to "forecasts" from 
identical initial conditions but with reduced errors in the troposphere. This reduction is achieved by periodically replacing selected components of the troposheric fields by observations (updating) during the prediction. We are particularly interested in performing "forecasts" in which only the zonal mean components of the prognostic variables are updated in the tropsphere.

The UCLA GCM is briefly described in Section 2. The 15 and 17 February cases are studied in Sections 3 and 4, respectively.

\section{Descriptions of the model and procedures for initialization and partial updates}

The main features of the UCLA GCM and initialization procedure used in the forecasts are in Mechoso et al. $(1985,1986)$. Here we summarize those aspects which are most relevant to this study.

The prognostic variables of the UCLA GCM are the horizontal velocity, the potential temperature, the water vapor mixing ratio, the ozone mixing ratio, the surface pressure, the planetary boundary layer (PBL) depth and the ground temperature. In the vertical, the model is based on a modified $\sigma$-coordinate (see Fig. 1). Isobaric surfaces are coordinate

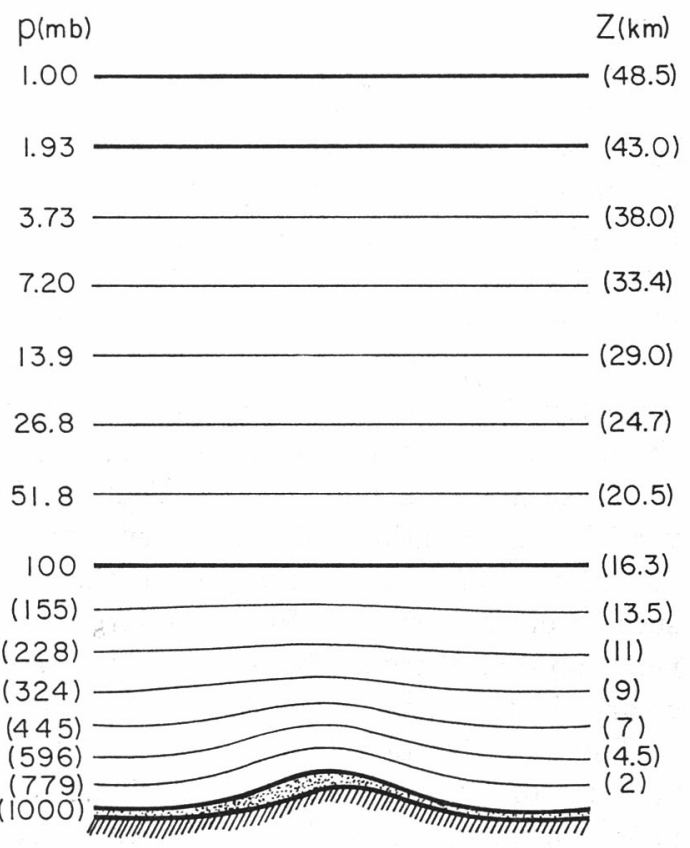

Fig. 1 Vertical structure of the UCLA GCM. surfaces above $100 \mathrm{mb}$. These include the model top at $1 \mathrm{mb}$ which is assumed to be a material surface. There are seven layers above $100 \mathrm{mb}$, resulting in a stratospheric resolution of about $4.5 \mathrm{~km}$. The lowest of the eight tropospheric layers represents the well-mixed variable-depth PBL in which turbulent processes are parameterized using bulk assumptions. The equations are horizontally discretized using a staggered latitude-longitude grid. For the experiments described in this paper, we used the low-resolution version $\left(4^{\circ}\right.$ of latitude by $5^{\circ}$ of longitude). In Mechoso et al. (1985), we also used the high-resolution version $\left(2.4^{\circ}\right.$ of latitude by $3^{\circ}$ of longitude).

The dataset used consists of the FGGE Level III-b analysis by the European Centre for Medium Range Weather Forecasts (ECMWF) below $10 \mathrm{mb}$, and the U.S. National Meteorological Center (NMC) global height and temperature fields above that level with winds computed from the geostrophic relation. All forecasts described in this paper started at 0000 GMT, but integrations started 12 hours earlier. To initiate an integration, the horizontal velocity, the potential temperature, the water vapor mixing ratio and the surface pressure were obtained from the dataset. The ozone mixing ratio was taken from an observed zonal mean climatology and the PBL depth was set equal to $50 \mathrm{mb}$ everywhere. After 12 hours of the integration, the predicted values of the horizontal velocity, potential temperature and surface pressure were replaced (updated) by the corresponding observed values in the dataset. Other prognostic variables were left unchanged except for water vapor mixing ratio, which was adjusted so as to preserve the relative humidity predicted by the model. In Mechoso et al. (1985) integrations were started five days before the forecast period and updates were performed every 6 or 12 hours depending on the frequency with which data are available. Reduction from 5 days to 12 hours in the length of the update cycle did not affect substantially the subsequent forecasts.

In the "forecasts" described in this paper, the updating procedure was continued every 
12 hours throughout the prediction for levels below $100 \mathrm{mb}$. For the level immediately above $100 \mathrm{mb}$, updated fields were a linear combination of those predicted and observed.

\section{The 15 February case}

We begin by describing selected observed features of the Northern Hemisphere circulation during the 10-day period starting from 0000 GMT 15 February and the corresponding forecasts.

The geopotential fields at $10 \mathrm{mb}$ for 0000 GMT 15, 20 and 25 February are given in Fig. 2. They show the splitting of the in-
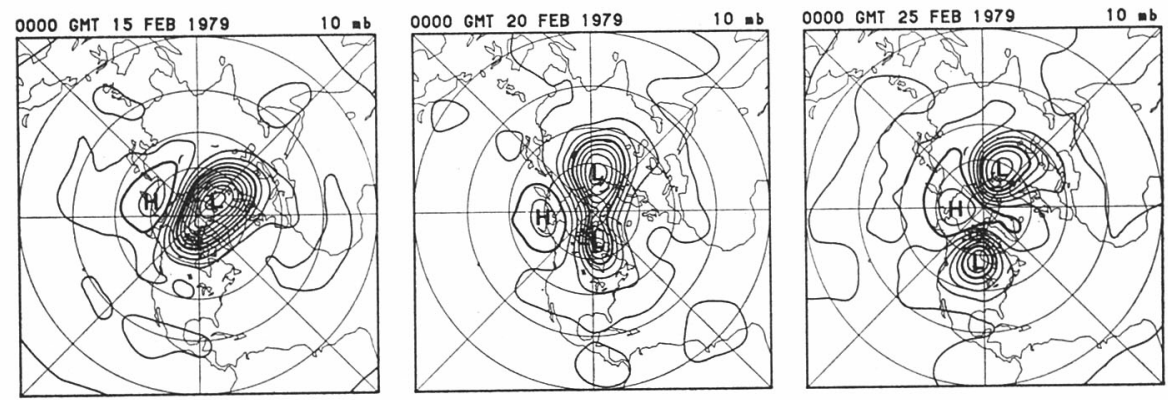

Fig. 2 Geopotential field at $10 \mathrm{mb}$ for 0000 GMT 15 (left), 20 (middle) and 25 (right) February 1979. The contour interval is $240 \mathrm{~m}$ and the 30,000 and $31,200 \mathrm{~m}$ contours are thicker.
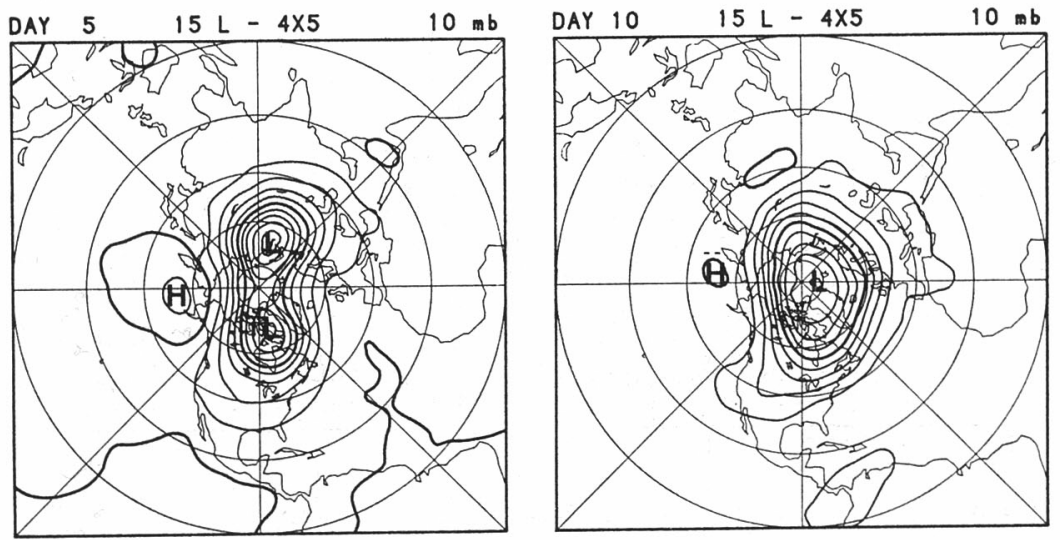

Fig. 3 5- (left) and 10-day (right) forecasts for $10 \mathrm{mb}$ from 15 February.
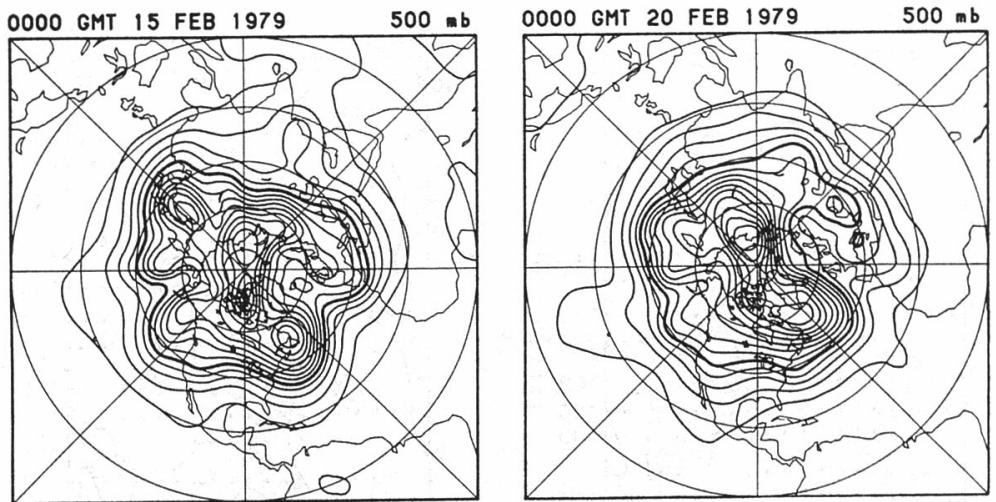

Fig. 4 Geopotential field at $500 \mathrm{mb}$ for $0000 \mathrm{GMT} 15$ (left) and 20 (middle) February 1979. The contour interval is $60 \mathrm{~m}$ and the $5,600 \mathrm{~m}$ contour is thicker. 
tense cyclonic vortex and poleward extension of the anticyclonic vortex that developed in association with the sudden warming. The 5- and 10-day forecasts for $10 \mathrm{mb}$ are in Fig. 3. They show that the initially well-predicted tendency towards vortex splitting reverses afterwards. This reversal is such that the flow becomes more zonal toward the end than at the beginning of the forecast, unlike the observed evolution.

The geopotential fields at $500 \mathrm{mb}$ for 0000 GMT 15 and 20 February are given in Fig. 4. They show the persistence of a welldefined zonal wavenumber 2 pattern at highlatitudes with ridges over the eastern North Atlantic Ocean and over the Bering Strait. The 5-day forecast (Fig. 5) shows a tendency to maintain the wavenumber 2 pattern but

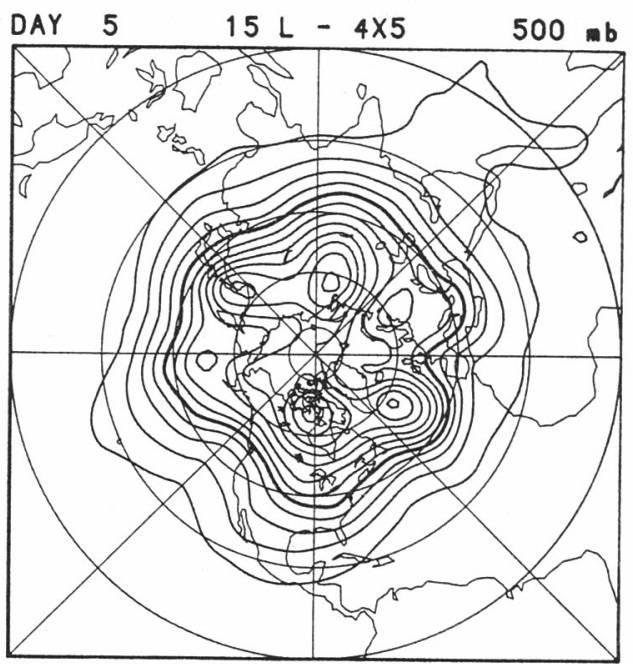

Fig. 5 5-day forecast for $500 \mathrm{mb}$ from 15 February.
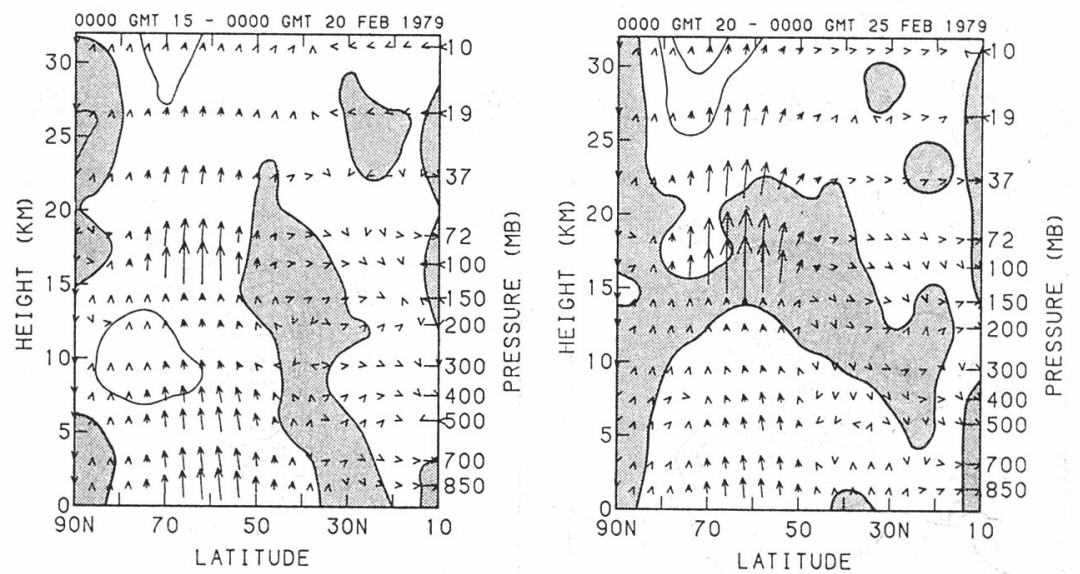

Fig. 6 E-P fluxes and their divergences averaged over 0000 GMT 15-0000 GMT 20 (left) and 0000 GMT 20-0000 GMT 25 (right) February 1979. The contour interval is 10 $\mathrm{ms}^{-1}$ day $^{-1}$ and positive values are shaded. Fluxes are multiplied by 10 above $100 \mathrm{mb}$.
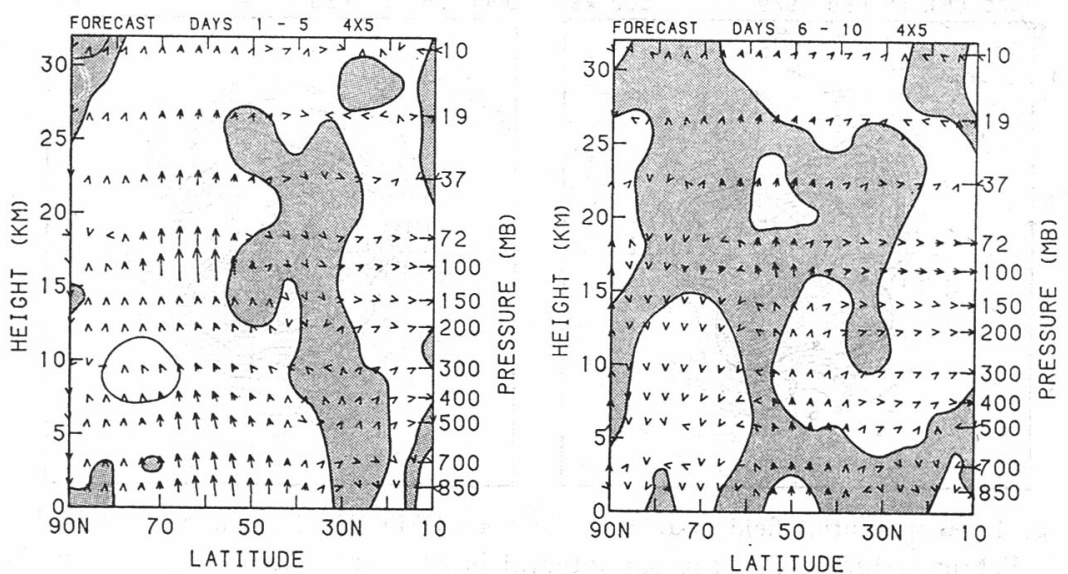

Fig. 7 As in Fig. 6, except for the forecast from 15 February. 
with a westward phase shift and general weakening at very high latitudes.

To show the effects of the eddies on the mean zonal flow, we used the transformed Eulerian mean diagnostic equations (Andrews and McIntyre, 1976; Palmer, 1981) as in Mechoso et al. (1985). Observed EliassenPalm (E-P) fluxes and their divergences for wavenumber 2 , averaged over the first and second halves of the period, are shown in Fig. 6. From about $50^{\circ}$ to $75^{\circ} \mathrm{N}$, fluxes are mostly vertical, indicating upward energy propagation. Fluxes in the stratosphere are stronger during the second than during the first half of the period while the opposite holds in the troposphere. Stronger flux convergences (decelerations) in the stratosphere occur during the second half of the period. Predicted E-P cross sections are in Fig. 7. They show that E-P fluxes in the forecast are weaker than observed during the first half of the period, notably north of $60^{\circ} \mathrm{N}$ in the troposphere, while they are much weaker than observed during the second half of the period.

As suggested by Butchart et al. (1982), the

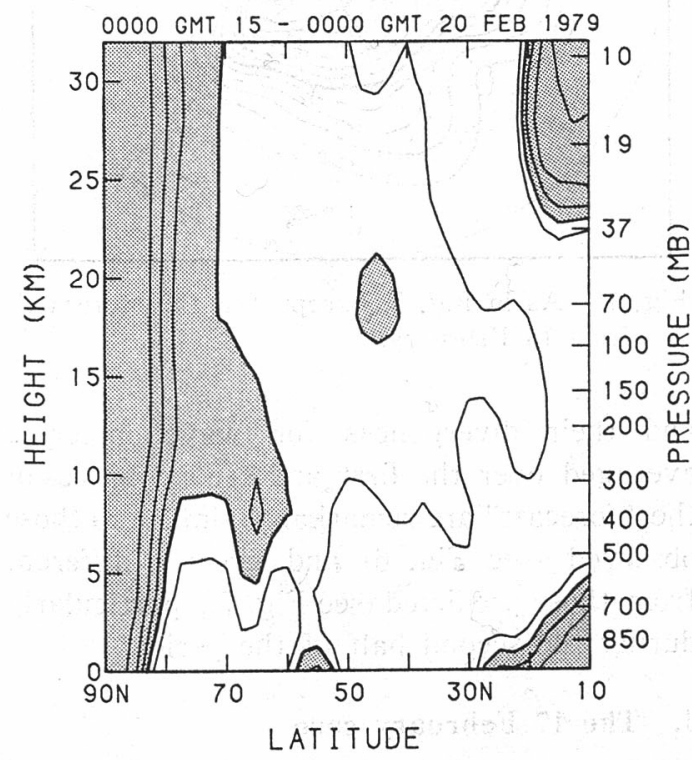

Fig. 8 Refractive index squared for wavenumber 2 averaged over 0000 GMT $15-0000$ GMT 20 February 1979. The contour interval is 50, negative values are shaded and values larger than 100 or lower than -150 are not contoured. refractive index is a useful diagnostic tool for planetary wave propagation even in unsteady cases, particularly when used in combination with the E-P flux. The average over the first half of the period of observed and predicted distributions of refractive index squared for wavenumber 2 are given in Figs. 8 and 9 , respectively. The observed distribution shows negative values at polar latitudes and a narrow region of low negative values in the upper troposphere between about $65^{\circ}$ and $70^{\circ} \mathrm{N}$. The predicted distribution also shows negative values in the polar region, but these extend further to lower latitudes in the upper troposphere than in the observation. There is also a narrow region of low negative values in the upper troposphere between about $60^{\circ}$ and $65^{\circ} \mathrm{N}$. The upper tropospheric zonal mean flow in the forecast is, therefore, less favorable than the observed for vertical propagation of wavenumber 2 in high latitudes. This is consistent with the differences pointed out above between the observed and predicted E-P fluxes.

We now describe the "forecast" from 0000 GMT 15 February 1979 in which zonal mean fields were periodically updated in the troposphere during the prediction following the procedure described in Section 2. The 5- and

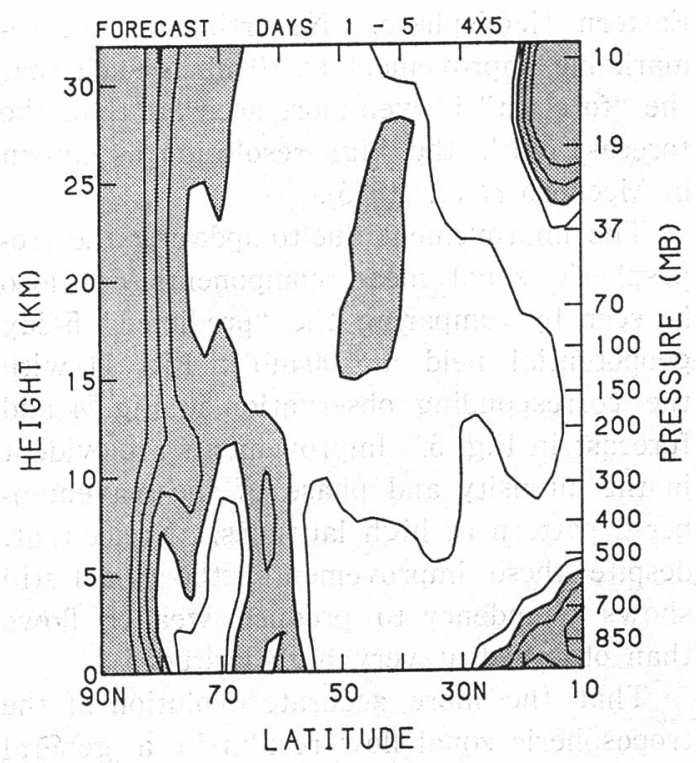

Fig. 9 As in Fig. 8, except for the forecast from 15 February. 

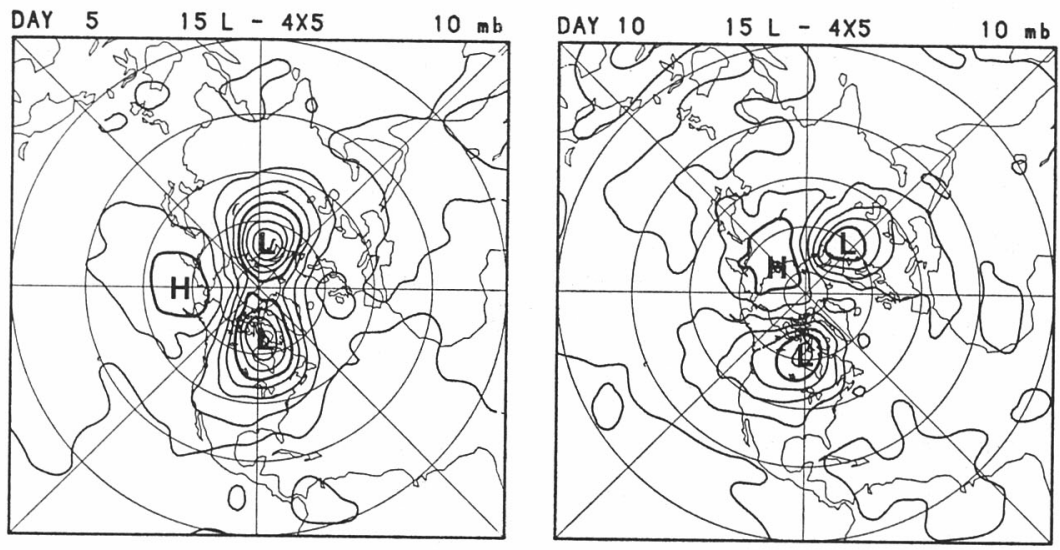

Fig. 10 As in Fig. 3, except for the "forecast" from 15 February in which the zonal mean components of selected fields are updated in the troposphere during the prediction.

10-day "forecasts" for $10 \mathrm{mb}$ are shown in Fig. 10 and can be compared with corresponding observations in Fig. 2 and forecasts in Fig. 3. It is immediately apparent that there is a drastic improvement, particularly at the end of the period. The splitting of the cyclonic vortex and the poleward extension of the anticyclonic vortex develop in the "forecast". There are, however, some quantitative differences with the corresponding observed field. The "predicted" intensity of lows and high are weaker than observed and there are visible phase differences in the Eastern Hemisphere. Nevertheless, the remarkable improvement at $10 \mathrm{mb}$ is such that the "forecast" is even more accurate than the forecast with the high resolution as shown in Mechoso et al. (1985).

The improvement due to updating the tropospheric zonal mean components can also be seen by comparing the "predicted" 5-day geopotential field at $500 \mathrm{mb}$ in Fig. 11 with the corresponding observation in Fig. 4 and forecast in Fig. 5. Improvements are evident in the intensity and phase of the wavenumber 2 pattern at high latitudes. Notice that, despite these improvements, the model still shows a tendency to produce weaker flows than observed at very high latitudes.

That the more accurate evolution of the tropospheric zonal flow results in a general improvement of wave, mean-flow interactions is clearly seen in Fig. 12. The E-P fluxes

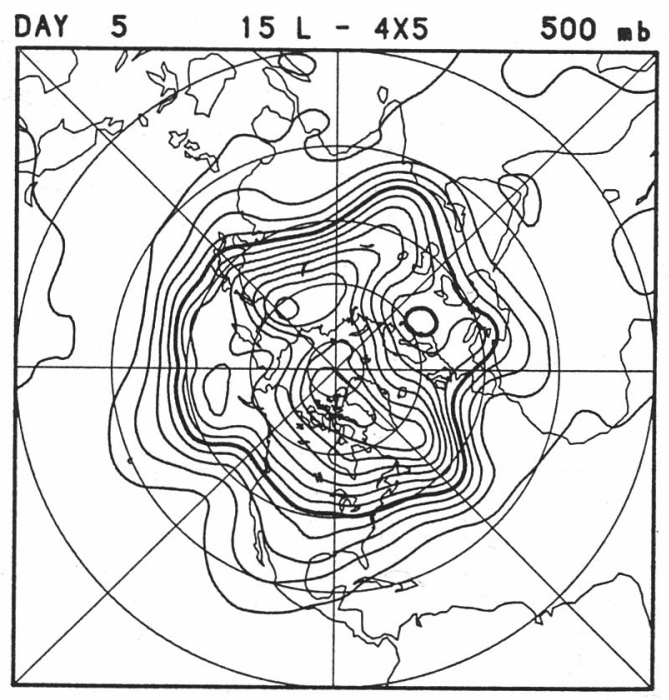

Fig. 11 As in Fig. 5, except for the "forecast" from 15 February.

and their divergences for wavenumber 2 averaged over the first and second halves of the "forecast" are remarkably similar to those observed (see Fig. 6) and greatly different from those predicted (see Fig. 7), particularly during the second half of the period.

\section{The 17 February case}

The geopotential fields at $10 \mathrm{mb}$ for 0000 GMT 17, 20 and 27 February are shown in Fig. 13. The 5- and 10-day forecasts for 10 $\mathrm{mb}$ from the former date are shown in Fig. 14. In this case, the forecast captures the 

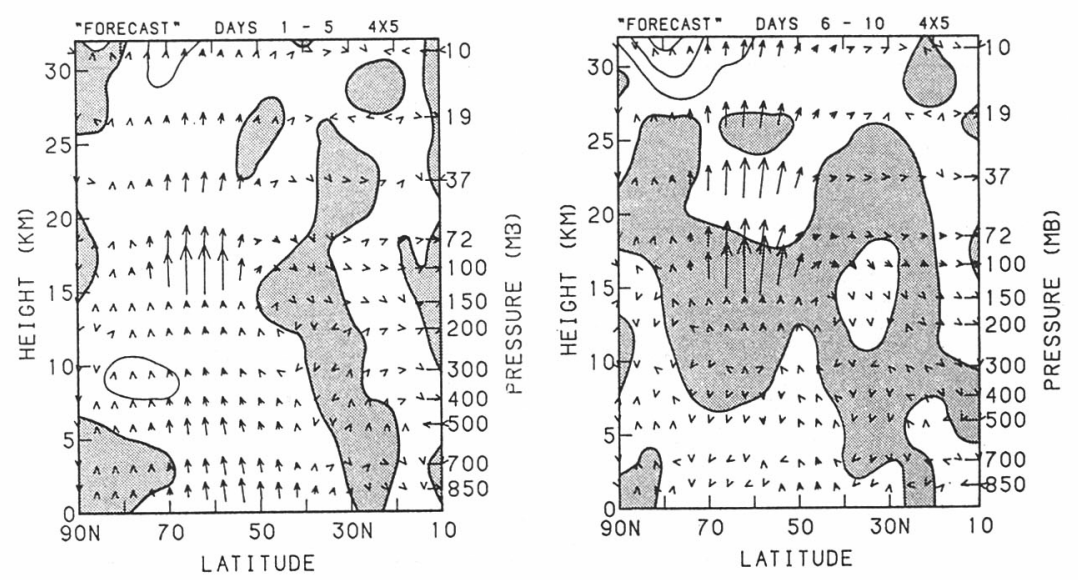

Fig. 12 As in and 7, except for the "forecast" from 15 February.
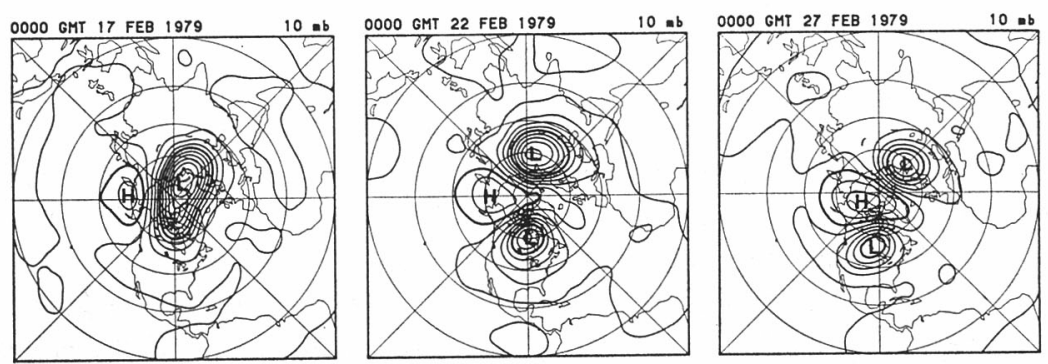

Fig. 13 Geopotential field at $10 \mathrm{mb}$ for 0000 GMT 17 (left), 22 (middle) and 27 (right) February 1979. Symbols are as in Fig. 2.
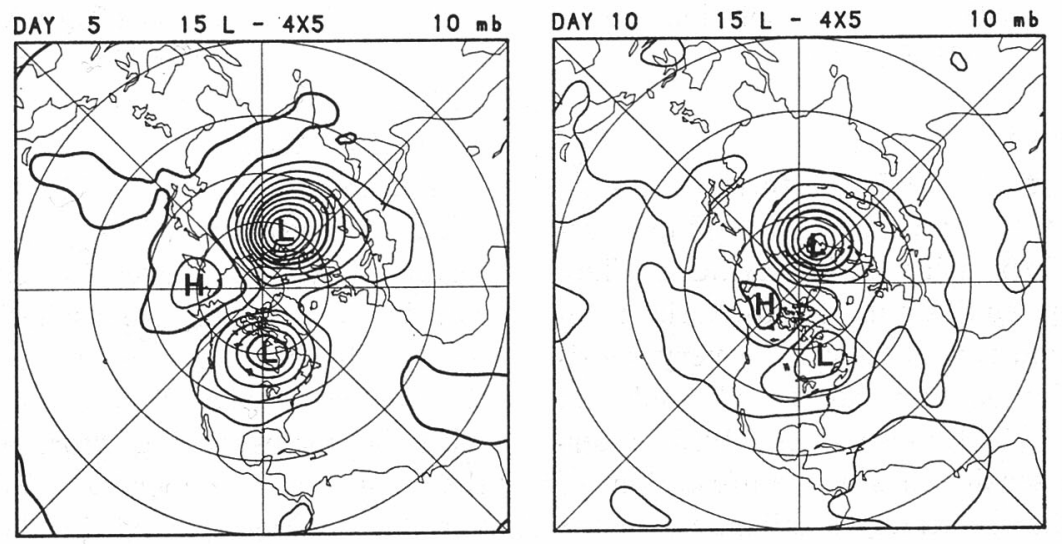

Fig. 14 Five- (left) and 10-day (right) forecasts for $10 \mathrm{mb}$ from 17 February.

breakdown of the polar vortex. At the end of the forecast period, however, the anticyclonic vortex is weaker than observed and one of the cyclonic vortices is far weaker than observed.

Corresponding 5- and 10-day "forecasts" for $10 \mathrm{mb}$ are in Fig. 15 and can be compared with the corresponding observations in
Fig. 13 and forecasts in Fig. 14. Overall, differences between forecast and "forecast" are less significant than those for the 15 February case. There is some improvement in the "predicted" intensity and location of the anticyclonic vortex and cyclonic vortex over northern North America. In addition, the separation between cyclonic vortices is 

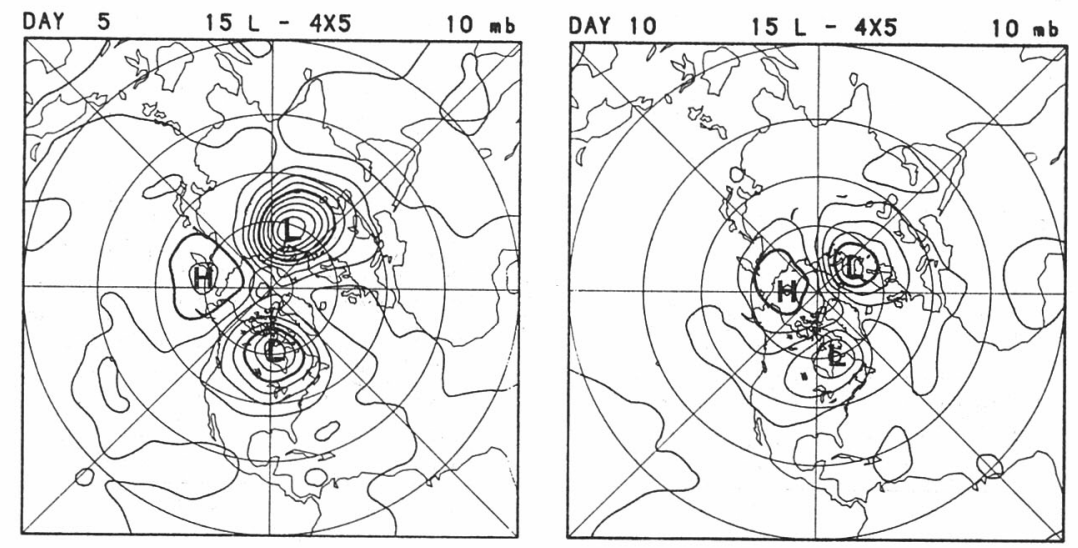

Fig. 15 As in Fig. 14, except for the "forecast" from 17 February in which the zonal mean components of selected fields are updated in the troposphere during the prediction.

somewhat clearer than it was in the forecast.

We also performed for this case a "forecast" with periodic updates of the ultra-long components of the fields (wavenumbers 1-3) in the troposphere. Results were closer to the observation than in the forecast as expected since we are now updating the forcing from the troposphere to the stratosphere. Interestingly, none of the two "forecasts" for this case were comparable in accuracy to the forecast with the high-resolution shown in Mechoso et al. (1985).

\section{Conclusions}

Mechoso et al. (1985) performed 10-day forecasts of the stratospheric warmings during winter 1979 by using the UCLA GCM. They obtained very successful stratospheric forecasts but found that these can be very sensitive to starting date and horizontal resolution of the model. Mechoso et al. (1985) interpreted these sensitivities as manifestations of the effects that relatively small errors in the predicted tropospheric zonal mean flow can have on the characteristics of upward wave energy propagation and wave, mean-flow interactions in the stratosphere. A reduction of these errors, by a later starting date or increased horizontal resolution, can result in a more accurate stratospheric forecast through a better tropospheric forecast.

The above interpretation is supported by the results of this study. The approach that was followed consisted in comparing forecasts for the major stratospheric warming of February 1979 with "forecasts" from identical initial conditions but with tropospheric evolution forced to follow closely the observed. This was obtained by periodically replacing selected components of the tropospheric fields by observations (updating) during the prediction. Two cases for the major sudden warming of February 1979 were considered with starting dates on 0000 GMT 15 and 17 February. All integrations were performed with the low-resolution, 15-layer version of the UCLA GCM.

The major finding of this study is that a drastic improvement of the stratospheric forecast can be achieved by reducing the errors in the predicted tropospheric zonal mean flow. This finding is obtained by comparing the forecast from 0000 GMT 15 February with the corresponding "forecast" in which the zonal mean components of the prognostic variables were updated in the troposphere. A less significant difference was found between the forecast from 17 February and the corresponding "forecast". This was expected, since there is much more room for improvement in the forecast from 15 February than in the forecast from 17 February.

The question remains as to why the forecast from 15 February is so much more sensitive to errors in the tropospheric zonal mean flow than that from 17 February. Observa- 
tions show that changes in zonal mean zonal velocities from 15 to 17 February are, in general, significantly smaller than those from 17 to 19 February. On the other hand, the corresponding changes in the first two days of the forecats from 15 and 17 February (as well as in other winter cases) are, in general, of comparable magnitude. This suggests that initial forecast errors have a strong systematic component due to deficiencies in the model and/or initialization procedure. It also suggests that this component is such that initial errors are larger for forecasts starting during periods in which the observed zonal mean flow is quasi-steady, as in the 15 February case.

\section{Acknowledgements}

We are grateful to Messrs. Joseph A. Spahr and John D. Farrara for programming assistance. Ms. Clara Wong typed the manuscript. This material is based upon work supported jointly by the National Science Foundation and the National Oceanic and Atmospheric Administration under Grant Number ATM 8218215 and the Naval Environmental Prediction Research Facility, Monterey, California, under Program Element 62759N, Project WF59-551, "Meteorological Models and Predictions", and by the National Science Foundation under Grant ATM 8515012.

\section{References}

Andrews, D. G. and M.E. McIntype, 1976: Planetary waves in horizontal and vertical shear: the generalized Eliassen-Palm relation and the mean zonal acceleration. J. Atmos. Sci., 33, 2031-2048.

Butchart, N., S. A. Clough, T. N. Palmer and P. J. Trevelyan, 1982: Simulations of an observed stratospheric warming with quasigeostrophic refractive index as a model diagnostic. Quart. J.R. Meteor. Soc., 108, 475-502.

Mechoso, C.R., K. Yamazaki, A. Kitoh and A. Arakawa, 1985: Numerical forecasts of stratospheric warming events during the winter of 1979. Mon. Wea. Rev., 113, 1015-1029.

, M. J. Suarez, K. Yamazaki, A. Kitoh and A. Arakawa, 1986: Numerical forecasts of tropospheric and stratospheric events during the winter of 1979: Sensitivity to the model's horizontal resolution and vertical extent, $A d v$. Geoph., 29, 375-413.

Miyakoda, K., R.F. Strickler and G.D. Hembree, 1970: Numerical simulation of the breakdown of a polar-night vortex in the stratosphere. $J$. Atmos. Sci., 27, 139-154.

Palmer, T.N., 1981: Diagnostic study of a wavenumber-2 stratospheric sudden warming in a transformed Eulerian-mean formalism. J. Atmos. Sci., 38, 844-855.

Simmons, A. J. and R. Strüfing, 1983: Numerical forecasts of stratospheric warming events using a model with a hybrid vertical coordinate. Quart. J. Roy. Meteor. Soc., 109, 81-111. 